\section{Fish-related Kounis syndrome: One entity, multiple triggers}

To the Editor,

Fish, shellfish, and seafood in general are often reported among the most active food inducers of Kounis syndrome (KS), although in the setting of fish poisoning, several molecular triggers and different pathogenic mechanisms may induce this clinical condition. The intriguing article by Gargiulo et al. (1) depicts a case of type II KS triggered by rotten tuna. The present case is an example of $\mathrm{KS}$ related to scombroid syndrome, resulting from the ingestion of histamine-fish poisoning, like spoiled raw fish, with subsequent conversion of the amino acid histidine into histamine by gram-negative bacteria, containing the enzyme histidine decarboxylase. In this situation, the pathogenic process is sustained by an anaphylactoid, non-Immunoglobulin $\mathrm{E}(\mathrm{IgE})$-mediated reaction, directly consequent to histamine toxicity, and mainly related to the consumption of rotten fish belonging to the Scombridae or Scomberesocidae spp. (such as tuna, mackerel, bonitos and sauries) and, less frequently, to the Clupeidae spp. (including anchovies, sardines, and herring) (2). In contrast to scombroid syndrome, Anisakis is another food-induced potential cause of KS, which is however sustained by an acute IgE-mediated generalized reaction, triggered by ingesting live larvae of Anisakis simplex, a parasitic nematode infesting raw, undercooked, or spoiled fish or seafood, which leads to mast cells activation, degranulation, and release of pre-formed or newly synthetized inflammatory mediators (3). Albeit underpinned by a different hypersensitivity process, these two fish-related clinical entities share a common denominator, which is related to pathophysiologic effects of histamine, including coronary vasoconstriction, platelet activation, intimal thickening, inflammatory cell modulation, and atherosclerotic plaque disruption (2). In addition, several fish proteins have been encountered as other potential triggers of $\mathrm{KS}$ related to IgE-mediated hypersensitivity reaction because of their intrinsic allergenic properties. Parvalbumins constitute a wide group of low-molecular-weight allergenic proteins, which are mainly expressed in fish muscles, as well as enolase and aldolase enzymes, which are involved in anaerobic glycolysis. Furthermore, the potential allergenic power of fish gelatin proteins should be clearly taken into account, considering their common use as additives in several foods, including jellies, beverages, lunch meats, and sweets (4). Finally, different tachykinins secreted from the salivary glands of octopus belonging to the Eledone spp. have been reported as biochemical inducers of $\mathrm{KS}$ as consequences of anaphylactoid reactions, due to their substance P-like vasoactive and inflammatory properties, as reported in a recently paper published by our research group (5). In conclusion, clinicians should be aware of the wide spectrum of biochemical triggers, effectors, and pathogenic mechanisms capable of inducing fish-related KS to timely start the most appropriate diagnostic strategies and therapeutic approaches.

\author{
Riccardo Scagliola1,2 (D), Claudio Brunelli1 (D) \\ ${ }^{1}$ Cardiovascular Disease Unit, IRCCS Ospedale Policlinico San \\ Martino and Department of Internal Medicine; Genova-Italy \\ 2Division of Cardiology, Department of Emergency, Cardinal \\ Massaia Hospital; Asti-Italy
}

\section{References}

1. Gargiulo C, De Martino S, Somaschini A, Cornara S, Crimi G. An intriguing case of acute coronary syndrome caused by rotten tuna. Anatol J Cardiol 2021; 25: 49-50. [Crossref]

2. Kounis NG. Kounis syndrome: an update on epidemiology, pathogenesis, diagnosis and therapeutic management. Clin Chem Lab Med 2016; 54: 1545-59. [Crossref]

3. Pravettoni V, Primavesi L, Piantanida M. Anisakis simplex: current knowledge. Eur Ann Allergy Clin Immunol 2012; 44: 150-6.

4. Kuehn A, Swoboda I, Arumugam K, Hilger C, Hentges F. Fish allergens at a glance: variable allergenicity of parvalbumins, the major fish allergens. Front Immunol 2014; 5: 179. [Crossref]

5. Scagliola R, Secchi G, Brunelli C. A Dangerous Octopus Fishing: Kounis Syndrome Following an Octopus Bite. J Coll Physicians Surg Pak 2020; 30: 675-6. [Crossref]

Address for Correspondence: Riccardo Scagliola, MD,

Cardiovascular Disease Unit, IRCCS Ospedale Policlinico San Martino and Department of Internal Medicine, University of Genova; Genova-Italy

Phone: +390141834626

E-mail: risca88@live.it

(C) Copyright 2021 by Turkish Society of Cardiology -

Available online at

www.anatoljcardiol.com

DOI:10.5152/AnatolJCardiol.2021.86579

\section{Author’s Reply}

To the Editor,

We read this letter with great interest, and we thank the authors for providing such a detailed pathophysiological explanation of the scombroid syndrome and fish-related KS. We agree with the authors on the importance of keeping in mind the triggers and effectors of KS in the context of acute coronary syndromes (ACSs) to timely apply the appropriate diagnostic and therapeutic tool.

In fact, ACSs are not always as straightforward as they may appear. In the case we reported, a patient with anaphylactoid type II KS presented with acute myocardial infarction likely due to coronary vasospasm causing distal embolization of a thrombus. We have highlighted the key role of unusual imaging techniques in the diagnostic work in specific subsets of patients with ACSs. Specifically, a timely cardiac magnetic resonance imaging helped us to reach the diagnosis of distal embolization, a condition uneasily discovered by other imaging techniques. In addition, OCT highlighted the traces of a residual thrombus in the right coronary artery, likely the origin of the embolus.

Every cardiologist should keep in mind the existence of KS and the wide spectrum of clinical manifestations to start the most appropriate diagnostic strategies and therapeutic approaches as soon as possible. 
Chiara Gargiulo (D), Silvana De Martino (D, Alberto Somaschini (D), Stefano Cornara (D, Gabriele Crimi1 (iD)

Coronary Care Unit and Laboratory of Clinical and Experimental Cardiology, Fondazione IRCCS Policlinico San Matteo; Pavia-Italy 1Division of Cardiology, Fondazione IRCCS Policlinico San Matteo, Pavia, Italy and Interventional Cardiology Unit, CardioThoraco Vascular Department (DICATOV) - IRCCS Ospedale Policlinico San Martino; Genova-Italy
Address for Correspondence: Stefano Cornara, MD,

Departments of Molecular Medicine, and Coronary Care Unit, Fondazione IRCCS Policlinico San Matteo and University of Pavia; Pavia-Italy

Phone: +390382507027

E-mail: stefano.cornara@gmail.com

(C) Copyright 2021 by Turkish Society of Cardiology - Available online at www.anatoljcardiol.com 